\title{
Upper Limb Exoskeleton Control for Isotropic Sensitivity of Human Arm
}

\author{
Rok Goljat, Tadej Petrič, and Jan Babič
}

\begin{abstract}
Most of today's assistive devices are controlled to provide uniform assistance irrespectively from the configuration of the human arm and the direction of the movement. We propose an innovative control method for arm exoskeletons that takes into account both of these parameters and compensates the anisotropic property of the force manipulability measure, intrinsic to the biomechanics of the human arm. To test our controller we designed a set of reaching tasks where the subjects had to carry two different loads to targets at five different locations and of two different sizes. Reaching times and trajectories were analysed for the evaluation of the controller. Through the analysis of the average reaching times we found that our method successfully enhances the motion while the analysis of the average maximal deviation from the ideal trajectories showed that our method does not induce any additional dynamic behaviour to the user.
\end{abstract}

\section{INTRODUCTION}

Robotic wearable devices such as exoskeletons are being developed to either augment the abilities of healthy individuals or to improve the condition of those with impaired physical abilities [1], [2]. A common approach to control such devices is to measure interaction dynamics and use the inverse dynamics models to either directly amplify the forces produced by the human muscles or to generate task dependent trajectories [3]. Although this approach efficiently augments human motion, it does not take into account the mechanical characteristics such as the highly anisotropic manipulability of the human arm. The aim of this paper is to propose a feed-forward control approach that augments the motion of the human arm by transforming the anisotropy of the arm manipulability [4], [5] in such a way that the axes become equal. Instead of amplifying the forces exerted by the human hand equally in all directions, our control approach produces forces in such a way that the manipulability ellipsoid becomes a circle.

\section{MATERIAL AND METHODS}

For the design of our control approach we considered two biomechanical parameters of the human arm: the manipulability measure which is based purely on the arm kinematics, and the mobility measure which takes into account both the kinematics and the dynamics of the arm.

The work presented in this paper was supported by the European Unions' Horizon 2020 research and innovation programme under grant agreemen No. 687662 - SPEXOR.

All authors are with the Automation, Biocybernetics and Robotics Department of the Jozef Stefan Institute, Ljubljana, Slovenija (email: rok.goljat@ijs.si, tadej.petric@ijs.si, jan.babic@ijs.si).

\section{A. Manipulability}

The manipulability measure takes the uncertainty of joint angles and transforms it to the uncertainty in end-point position. By assuming that the joint sensors are noisy with variance $\sigma^{2}$ and independent from each other then the covariance of uncertainty is transformed from joint space to covariance of uncertainty at the end-point:

$$
\operatorname{Cov}(\Delta \mathbf{x})=\mathbf{J} \operatorname{Cov}(\Delta \mathbf{q}) \mathbf{J}^{T}=\sigma^{2} \mathbf{M} .
$$

Here, $\Delta \mathbf{x}$ and $\Delta \mathbf{q}$ are the end-point and joint uncertainties and $\mathbf{J}$ is the Jacobian of the current arm configuration. In (1), the matrix

$$
\mathbf{M}=\mathbf{J J}^{T}
$$

represents the manipulability matrix [6] that shapes the independent joint noise into Cartesian end-point noise.

\section{B. Mobility}

The mobility measure is based on the instantaneous response of the arm to dynamic perturbations [7]. If the inertial matrix $\mathbf{H}$ of the arm is known, we can then define the mobility measure as

$$
\mathbf{W}=\mathbf{J H}^{-1} \mathbf{J}^{T},
$$

where $\mathbf{W}$ represents the end-point mobility matrix [8]. Because mobility also includes the inertia of the arm, it can be considered as a more precise measure of arm sensitivity.

\section{Control Method}

The eigenvectors of mobility and manipulability matrices represent directional sensitivity of the arm [7]. Specifically, it is the easiest to control the end-effector position and sense perturbations along the axis of the minor eigenvector and the hardest in the direction of the major eigenvector. If we invert the sensitivity matrices, than they relate to the joint torque and end-effector force. In this case, the major eigenvector points in the direction where it is the easiest to exert the end-effector force and the minor eigenvector points in the direction where it is the hardest to exert the end-effector force. To counteract these anisotropic properties we propose a novel control method for arm exoskeletons that transforms the anisotropic sensitivity of the arm to the isotropic sensitivity. Using singular value decomposition, the sensitivity matrices can be represented as ellipses with the major and minor axes representing the major and minor eigenvectors.

To augment the user exerted force and obtain isotropic sensitivity, the assistive force $\boldsymbol{F}_{a}$ needs to equal the user 

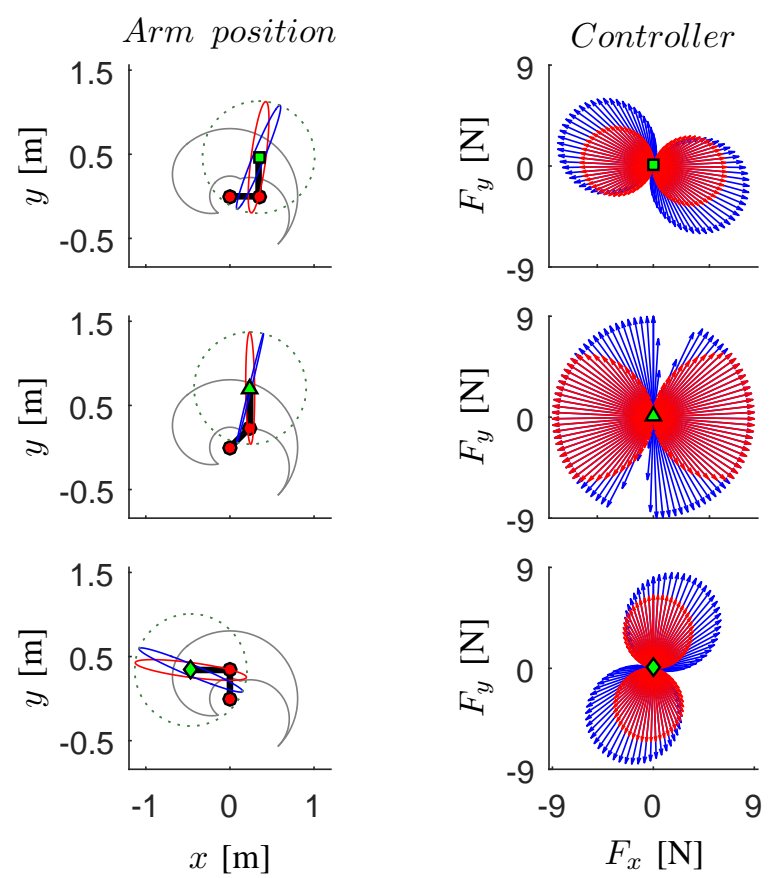

Fig. 1. Plots in the left column show the inverted manipulability (blue) and mobility (red) ellipsoids for tree different positions of the arm inside the workspace (black). The dotted lines show the normalized ellipsoids. Plots in the right column show the assistive forces of the proposed controller for the corresponding configurations in the left column. The arrows represent the assistive forces for the manipulability measure (blue) and the mobility measure (red).

exerted force $\boldsymbol{F}_{u}$ amplified by the ratio between ellipse major axis $M_{a x i s}$ and the projection of the user force $F_{a x i s}$ on the ellipse (4).

$$
\boldsymbol{F}_{a}=\left(\frac{\left\|M_{\text {axis }}\right\|}{\left\|F_{\text {axis }}\right\|}-1\right) \boldsymbol{F}_{u}
$$

\section{EXPERIMENTS AND RESULTS}

We implemented the proposed controller in a simulation environment and compared its performance for both mobility and manipulability measures. For the manipulability measure, the controller was also experimentally evaluated on 5 subjects.

Fig. 1 shows the forces generated by the controller if the user exerted $1 \mathrm{~N}$ forces in three different positions of the arm. Due to the thin shape of the ellipse on the edges of the workspace, larger assistive forces were exerted there than in the central region of the workspace. For the safety reasons, the maximum assistive force was limited to $9 \mathrm{~N}$.

Subjects performed unassisted and assisted load carrying motions to five different targets of two sizes $(20 \mathrm{~mm}, 5$ $\mathrm{mm}$ ). The motions were performed on a transverse plane at the shoulder height. The experiment consisted of three consecutive sessions. In the first session, the subjects were unassisted and had to move a $3 \mathrm{~kg}$ object (baseline session). In the second session, the subjects were also unassisted
TABLE I: Experimental results

\begin{tabular}{ccc}
\hline \hline Trial (target size) & Average time [s] & Average deviation [m] \\
\hline Baseline (large) & 0.51 & 0.024 \\
Heavy (large) & 0.76 & 0.028 \\
Assisted (large) & 0.58 & 0.018 \\
Baseline (small) & 0.98 & 0.030 \\
Heavy (small) & 1.26 & 0.028 \\
Assisted (small) & 1.05 & 0.025 \\
\hline \hline
\end{tabular}

but had to move a heavier object weighting $30 \mathrm{~kg}$ (heavy session). In the final session the subjects had to move the $30 \mathrm{~kg}$ object but were assisted by the proposed controller (assisted session).

Table I shows the average reaching times and average maximum deviations of motions performed by subjects during the three sessions and for both target sizes. When the subjects were asked to move a heavy load, their average reaching times were larger than during the baseline session. When they were asked to move a heavy load while being assisted by the controller, their average reaching times were close to the times during the baseline session. Decreasing the target size increased the overall time of the movements but did not have any notable effect on the motion trajectory.

\section{CONCLUSIONS}

We developed and evaluated a novel controller to augment the motion of the human arm based on its manipulability and mobility measures. By simulations and an experimental study we demonstrated that the proposed control method successfully augments human capabilities without introducing any additional disturbances to the human motion. In the future we plan to carry out an extensive experimental study involving both manipulability and mobility measures and further expand the sensitivity model to generalize it for more complex tasks.

\section{REFERENCES}

[1] A. M. Dollar and H. Herr, "Lower extremity exoskeletons and active orthoses: Challenges and state-of-the-art," IEEE Transactions on Robotics, vol. 24, pp. 144-158, 2008.

[2] L. Peternel, T. Noda, T. Petrič, A. Ude, J. Morimoto, and J. Babič, "Adaptive control of exoskeleton robots for periodic assistive behaviours based on emg feedback minimisation," PLoS ONE, vol. 11, no. 2, 02 2016.

[3] L. Peternel, T. Petrič, E. Oztop, and J. Babič, "Teaching robots to cooperate with humans in dynamic manipulation tasks based on multimodal human-in-the-loop approach," Autonomous Robots, vol. 36, no. 1, pp. 123-136, 2013.

[4] I. Cos, N. Belanger, and P. Cisek, "The influence of predicted arm biomechanics on decision making," Journal of Neurophysiology, vol. 105, no. 6, pp. 3022-3033, jun 2011.

[5] M. Yamashita, "Robotic Rehabilitation System for Human Upper Limbs Using Guide Control and Manipulability Ellipsoid Prediction," Procedia Technology, vol. 15, pp. 559-565, 2014.

[6] T. Yoshikawa, Foundations of Robotics: Analysis and Control. Cambridge, MA, USA: MIT Press, 1990.

[7] P. N. Sabes and M. I. Jordan, "Obstacle avoidance and a perturbation sensitivity model for motor planning." The Journal of neuroscience : the official journal of the Society for Neuroscience, vol. 17, no. 18, pp. 7119-28, sep 1997.

[8] N. Hogan, "Impedance control: An approach to manipulation: Part iiimplementation," Journal of dynamic systems, measurement, and control, vol. 107, no. 1, pp. 8-16, 1985. 